\title{
Estudo Comparativo de Sete Diferentes Modelos Estatísticos para a Característica Ganho de Peso em Bovinos de Corte
}

\author{
Henrique Dorneles Fernandes ${ }^{1}$, Gilka Barbosa Ferreira ${ }^{2}$
}

\begin{abstract}
RESUMO - Foram analisadas uni e bivariadamente as características ganho de peso do nascer à desmama e ganho de peso pós-desmama de 4883 animais, filhos de 328 touros da raça Charolês, com o objetivo de comparar diferentes modelos ao considerado mais completo, porém de mais difícil aplicabilidade. Os modelos estudados foram o MA1, modelo animal que inclui os efeitos genético direto do animal e genético materno; MA2, modelo animal que inclui o efeito aditivo direto do animal e o efeito de ambiente permanente; MA3, que inclui os três efeitos já citados (considerado o "melhor"); MA4, modelo animal que inclui apenas o efeito aditivo direto; MTV, modelo touro-vaca que inclui o efeito genético direto do pai, além dos efeitos genéticos materno e de ambiente permanente; MT1, modelo touro com efeito genético aditivo direto do pai e efeito de ambiente permanente; e MT2, modelo touro com apenas efeito direto do pai. Com base nos resultados, observou-se diferença estatística somente entre os modelos MA4 e MT2, em relação ao MA3, para o ganho de peso do nascer à desmama. Concluiu-se que, para a característica pré-desmama, a inclusão de pelo menos um dos efeitos (genético materno ou ambiental) foi indicada, enquanto para a pós-desmama até mesmo modelos mais simples como o MA4 e o MT2 podem ser utilizados para substituir o modelo mais complexo (MA3). As análises bivariadas são indicadas, principalmente por apresentarem as correlações genéticas entre as características.
\end{abstract}

Palavras-chave: efeito ambiental permanente, efeito materno, ganho de peso

\section{Comparative Study of Seven Different Statistical Models for Weight Gain in Beef Cattle}

\begin{abstract}
Using a univariate and a multitrait analyses, the traits weight gain from birth to weaning (WGBW) and weight gain from weaning to yearling (WGWY) were analyzed on 4883 Charolais beef cattle, sons of 328 sires, to compare different models with the most complete model, although with more difficult applicability. The models were the MA1, animal model which included the direct effect of the animal and the genetic maternal effect; MA2, animal model which included the direct effect of the animal and the permanent environmental effect; MA3, which included the three effect (considered "the best"); MA4, animal model which included only the animal direct effect; MTV, the sire-dam model which included the direct genetic effect of the sire, plus the maternal genetic effect and the permanent environmental effect; MT1, sire model which included the genetic effect of the sire and the permanent environmental effect and the MT2, sire model which included only the direct genetic effect of the sire. The results showed that there were statistical differences among the models MA4 and MT2, comparing with the MA3 but only for WGBW. It was concluded that for WGBW the inclusion in the model of at least one effect (genetic maternal or environmental) was indicated, while for WGWY simple models, as the MA4 or MT2, could be utilized in substitution to the most complex models (MA3). The multitrait analyses were indicated, mainly because it presented the genetic correlation among the traits
\end{abstract}

Key Words: permanent environmental effect, maternal effect, weight gain

\section{Introdução}

As características de crescimento em bovinos de corte são muito importantes em qualquer programa de seleção. O conhecimento da influência materna em características pré e pós-desmama é fundamental, quando se objetiva a formulação de Programas de Melhoramento Genético. Quando se analisam dados de bovinos de corte, não somente é necessária a diferenciação de fatores genéticos e ambientais, mas também a parte genética tem que ser separada em efeitos diretos e maternos. O problema principal para entender a variação genética é a relação entre efeito direto e materno, pois o antagonismo entre eles poderia ter conseqüências para o Programa de Melhoramento.

Os efeitos maternos nos animais têm sido estudados em anos recentes e muitos destes estudos têm sugerido correlação genética negativa entre os efeitos diretos e maternos para peso à desmama. As bases biométricas para análise de efeito materno

\footnotetext{
${ }^{1}$ Aluno do curso de Pós-graduação em Zootecnia da Universidade Federal de Santa Maria, RS. E.mail: a9960280@alunop.ufsm.br Autor para correspondências.

2 Professor Adjunto do Depto. de Zootecnia da UFSM, 97105-900, Santa Maria, RS. E.mail: gbbf@ccr.ufsm.br
} 
foram desenvolvidas por DICKERSON (1947) e KOCH e CLARK (1955). Mais tarde, WILLHAM (1963) expressou os efeitos maternos em termos de modelos lineares e WILLHAM (1972) desenvolveu princípios de análise biométrica para efeito diretos e maternos e também considerou a influência dos efeitos da avó.

THOMPSON (1976) desenvolveu uma aproximação de máxima verossimilhança, baseada em uma análise de variância multivariada, para estimativa de variância genética materna. Foi descrito como o método pode ser aplicado para a estimativa da variância genética materna e como pode ser modificado, quando os pais são selecionados pelos seus valores fenotípicos.

No estudo de MEYER et al. (1993), estimativas de componentes de variância, devido aos efeitos diretos e maternos para peso ao nascer, desmama, sobreano e peso ao final, foram obtidas com um modelo animal. Ambos os efeitos, genético materno e efeito ambiental permanente, afetaram os diferentes pesos, com exceção do peso final. Estimativas de correlação genética entre efeito direto e materno para peso à desmama foram negativas e de moderadas a alta.

Usando o modelo descrito por MEYER et al. (1993), SWALVE (1993) encontrou, para peso ao nascer, peso à desmama e sobreano, as estimativas de 0,$33 ; 0,35 ;$ e 0,37 , respectivamente, para herdabilidades diretas, e 0,$07 ; 0,18$; e 0,11 para herdabilidades maternas. Correlações negativas foram encontradas entre efeitos genéticos direto e materno para pesos à desmama e sobreano, em um intervalo de $-0,39$ a $-0,22$.

Na revisão de MOHIUDDIN (1993), as estimativas de parâmetros genéticos e fenotípicos para peso ao nascer, desmama, sobreano, medida muscular e marmoreio foram amplamente discutidas. As estimativas de herdabilidade para peso ao nascer, desmama e sobreano foram, em média, de 0,46; 0,39; e 0,24 para machos; 0,$26 ; 0,23$; e 0,20 para as fêmeas; e 0,$49 ; 0,48$; e 0,41 para os dois sexos combinados. As médias correspondentes para a medida muscular e o depósito de gordura foram de 0,24 e 0,$31 ; 0,28$ e 0,24; e 0,57 e 0,33 , respectivamente.

KOOTS et al. (1994a), em uma revisão abrangendo 45 anos (1946 a 1991), encontraram um total de 1655 estimativas de herdabilidade envolvendo 70 características e 287 trabalhos científicos. Esses autores observaram que as herdabilidades derivadas de dados experimentais, em média, não diferiram entre si para as características de peso. Verificaram ainda que as estimativas de herdabilidade foram afetadas pelas médias e desvios-padrão fenotípicos da população da qual foram estimadas. Na análise de covariância, foram encontradas estimativas de herdabilidade positivamente correlacionadas com a de outras características.

Em outro estudo, KOOTS et al. (1994b), utilizando informações oriundas de trabalhos publicados entre 1940 e 1991, analisaram as estimativas de correlação genéticas e fenotípicas entre diversas características. Nesse caso, uma análise ponderada de quadrados mínimos estimados para cada parâmetro estudado revelou que diversos fatores os influenciaram significativamente $(\mathrm{P}<0,10)$, incluindo a raça, o ano, o sexo e o rebanho. Estimativas de correlações genéticas foram comparadas com a correspondente estimativa de herdabilidade e estimativas de correlações fenotípicas e com as outras estimativas de correlações genéticas derivadas dos mesmos dados mais freqüentemente do que seria esperado apenas por chance. As implicações destas associações entre parâmetros foram discutidas com o objetivo de obter variância-covariância positiva-definida requerida nos programas de Melhoramento Animal.

A variância e a covariância para peso ao nascer, ganho do nascimento ao desmame e peso aos 205 dias foram estimadas em um estudo de JOHNSON et al. (1992), usando dois modelos animais distintos: um modelo considerando touro e vaca e um modelo de touro e avô materno, com e sem a inclusão da relação de parentesco. A inversa da matriz de parentesco $\left(\mathrm{A}^{-1}\right)$ foi calculada usando o método de HENDERSON (1976) para populações não consangüíneas. Constatou-se que, incluindo a matriz de parentesco, foram observadas estimativas de parâmetros genéticos mais altas para os efeitos diretos do que quando a matriz de parentesco não foi incluída. Os componentes de variância para vaca, avô materno e erro experimental foram similares com a inclusão ou não da $\mathrm{A}^{-1}$. VAN VLECK e HUDSON (1982), usando o método III de Henderson para a característica produção de leite, em vacas, relataram que as estimativas dos componentes de variância de touro aumentaram de 2 a 5\%, quando a relação de parentesco foi incluída.

BERTRAND e BENYSHEK (1987) usaram um modelo de touro-vaca e de touro e avô materno para estimar os componentes de variância para peso ao nascer e peso à desmama de animais das raças Limousin e Brangus. Foi determinado que, usando um modelo touro-vaca, a magnitude do componente de 
1342 Rev. bras. zootec.

variância para touro foi maior do que quando foi usado o modelo touro e avô materno. As correlações entre efeitos genéticos diretos e maternos foram negativas. As variâncias materno-ambiental permanentes foram de 89,38 e $21 \%$ da variância genética materna para peso ao nascer e à desmama, na raça Limousin, e peso à desmama, na raça Brangus, respectivamente. Pequena estimativa de variância ambiental permanente foi observada para Brangus no peso ao nascer.

MERCADANTE e LÔBO (1997) encontraram diferença significativa $(\mathrm{P}<0,05)$ entre o modelo animal que incluiu efeito genético aditivo direto do animal, efeito de ambiente permanente e efeito do genótipo da mãe em relação aos modelos que excluíram o efeito ambiental ou materno ou ambos, para característica de peso à desmama (240 dias).

Em anos recentes, o aumento da capacidade computacional tem facilitado o uso de modelos estatísticos mais sofisticados e detalhados para se estimarem componentes de variância e valores genéticos dos indivíduos. Além dos modelos de touros, é possível desenvolver, atualmente, outros como os modelos animais com e sem efeito materno e o modelo touro-vaca. Esses modelos podem ser comparados com os modelos touro e animal. A relação de parentesco entre animais pode ser incluída e as comparações entre os diferentes modelos estatísticos podem ser feitas para determinar a viabilidade dos mesmos. Essas comparações são importantes, pois para as estimativas de parâmetros genéticos de grande volume de dados é bem mais trabalhosa e demorada a utilização do modelo animal e, nestes casos, modelos mais simples são a única opção.

Portanto, é importante saber como as estimativas obtidas por meio destes modelos mais simples diferem daquelas oriundas de um mais completo, como o modelo animal incluindo efeito aditivo genético materno e efeito de ambiente permanente. Além disso, é importante analisar como se ordenam as estimativas de valores genéticos dos indivíduos nos diferentes modelos.

O objetivo principal deste trabalho foi comparar estimativas de componentes de variância e Diferenças Esperada na Progênie (DEP) para ganho de peso do nascer à desmama e ganho de peso pósdesmama de animais da raça Charolês, criados no Estado do Rio Grande do Sul, usando diferentes modelos estatísticos.

\section{Material e Métodos}

Foram utilizados, neste trabalho, dados referentes a 4883 animais, filhos de 328 touros da raça Charolês, coletados pelo Programa de Melhoramento de Bovinos (PROMEBO) de Pelotas, em rebanhos de diferentes regiões do Estado do Rio Grande do Sul, no período de 1983 a 1995.

Primeiramente os dados foram organizados utilizando o programa Statistical Analysis System (SAS, 1985), para verificar ou detectar eventuais problemas de digitação que pudessem comprometer o estudo. Foram eliminados registros extremos, ou seja, valores acima e abaixo de um e meio desvios-padrão em relação à média.

Foram estudadas as características ganho de peso do nascer à desmama (GPND) e ganho de peso pósdesmama - GPPD (550 dias).

As características foram analisadas univariada e bivariadamente, utilizando-se sete modelos, sendo que em todos foi incluído efeito fixo, de grupo contemporâneo do nascer à desmama, que considera: criador, rebanho, ano, estação, sexo, código alimentar e data juliana de pesagem, para a primeira característica (GPND), e grupo contemporâneo pósdesmama para a segunda característica (GPPD), que considera, além dos já citados, o sexo, o código alimentar e a data juliana de pesagem coletados novamente após o desmame.

Os modelos estudados, os quais constam na Tabela 1, foram: MA1, modelo animal que incluiu o efeito genético aditivo direto do animal e o efeito genético materno; MA2, modelo animal que incluiu, além do efeito direto do animal, o efeito de ambiente permanente, representado pela capacidade leiteira da mãe; MA3, modelo animal mais completo, que incluiu os três efeitos já citados; MA4, modelo animal que incluiu apenas o efeito genético direto do animal; MTV, modelo touro-vaca que incluiu o efeito genético aditivo direto do pai, porém utiliza a matriz inversa de parentesco $\left(\mathrm{A}^{-1}\right)$ envolvendo todos os animais, além de incluir os efeitos genético materno e de ambiente permanente; MT1, modelo touro que incluiu o efeito genético direto do pai, a $\mathrm{A}^{-1}$ contendo informações sobre os touros e, ainda, o efeito de ambiente permanente; e MT2, modelo touro com a inclusão apenas do efeito genético aditivo direto do pai.

Para desmembrar as estimativas dos componentes de (co) variância dos modelos touro e touro-vaca, segundo FERREIRA (1999), as seguintes composições foram usadas: 
Tabela 1 - Demonstrativo dos modelos utilizados no trabalho

Table 1 - Different models used in this work

\begin{tabular}{|c|c|c|c|c|}
\hline $\begin{array}{l}\text { Efeito } \& \\
\qquad A c\end{array}$ & $\begin{array}{l}\text { genético aditivo direto } \\
\text { do animal } \\
\text { Additive direct effect } \\
\text { of the animal }\end{array}$ & $\begin{array}{c}\text { Efeito genético aditivo direto } \\
\text { do pai } \\
\text { Additive directeffect } \\
\text { of the sire }\end{array}$ & $\begin{array}{l}\text { Efeito genético } \\
\text { materno } \\
\text { Maternal genetic } \\
\text { effect } \\
\end{array}$ & $\begin{array}{c}\text { Efeito de ambiente } \\
\text { permanente } \\
\text { Permanent } \\
\text { environmental effect }\end{array}$ \\
\hline \multicolumn{5}{|l|}{$\overline{\text { MA1 }}$} \\
\hline Modelo Animal & $\mathrm{X}$ & & $X$ & \\
\hline \multicolumn{5}{|l|}{ Animal model } \\
\hline Modelo Animal & $\mathrm{X}$ & & & $\mathrm{X}$ \\
\hline \multicolumn{5}{|l|}{$\begin{array}{l}\text { Animal model } \\
\text { MA3 }\end{array}$} \\
\hline Modelo Animal & $\mathrm{X}$ & & $\mathrm{X}$ & $X$ \\
\hline \multicolumn{5}{|l|}{ Animal model } \\
\hline \multirow{2}{*}{\multicolumn{5}{|c|}{$\begin{array}{l}\text { Modelo Animal } \\
\text { Animal model }\end{array}$}} \\
\hline & \\
\hline \multirow{2}{*}{\multicolumn{5}{|c|}{$\begin{array}{l}\text { Modelo touro-vaca } \\
\text { Sire-dam model }\end{array}$}} \\
\hline & & & & \\
\hline \multicolumn{5}{|l|}{ MT1 } \\
\hline Modelo touro & & $\mathrm{X}$ & & $\mathrm{X}$ \\
\hline \multicolumn{5}{|l|}{ Sire model } \\
\hline \multicolumn{5}{|l|}{ MT2 } \\
\hline Modelo Touro & & $\mathrm{X}$ & & \\
\hline
\end{tabular}

Modelo touro:

$\sigma_{\mathrm{s}}^{2}=(1 / 4) \sigma_{\mathrm{g}}^{2}$;

$\sigma_{\mathrm{e}}^{2}=(3 / 4) \sigma_{\mathrm{g}}^{2}+\sigma_{\mathrm{m}}^{2}+\sigma_{\mathrm{gm}}+\sigma_{\mathrm{p}}^{2}+\sigma_{\mathrm{te}}^{2}$;

Modelo touro-vaca:

$\sigma_{\mathrm{s}}^{2}=(1 / 4) \sigma_{\mathrm{g}}^{2}$;

$\sigma_{\mathrm{d}}^{2}=(1 / 4) \sigma_{\mathrm{g}}^{2}+\sigma_{\mathrm{m}}^{2}+\sigma_{\mathrm{gm}}$;

$\sigma_{\mathrm{sd}}=(1 / 4) \sigma_{\mathrm{g}}^{2}+(1 / 2) \sigma_{\mathrm{gm}}$

$\sigma_{\mathrm{e}}^{2}=(1 / 2) \sigma_{\mathrm{g}}^{2}+\sigma_{\mathrm{te}}^{2}$;

em que

$\sigma^{2}{ }_{\mathrm{S}}$ é a variância dos efeitos dos touros;

$\sigma_{\mathrm{g}}^{2}$ é a variância genética;

$\sigma_{\mathrm{d}}^{2}$ é a variância dos efeitos das vacas;

$\sigma_{\text {sd }}$ é a covariância entre os efeitos dos touros e das

vacas;

$\sigma_{\mathrm{m}}^{2}$ é a variância dos efeitos maternos;

$\sigma_{\mathrm{gm}}$ é a covariância entre os efeitos genéticos diretos e maternos;

$\sigma^{2}$ é a variância dos efeitos ambientais permanentes; $\sigma_{\text {te }}^{2}$ é a variância dos efeitos ambientais temporários; e $\sigma^{2}$ é a variância dos efeitos ambientais.

As estimativas de componentes de (co) variância e de DEPs (diferença esperada na progênie) foram obtidas por intermédio do programa computacional
MTDFREML (Multiple Trait Derivative-Free Restricted Maximum Likelihood), de BOLDMAN et al. (1995).

$\mathrm{O}$ critério de convergência utilizado foi $10^{-6}$, sendo que eram feitas três repetições partindo dos valores obtidos na análise anterior para obter maior confiabilidade nos resultados.

Foram feitas comparações, de posse dos resultados finais dos componentes de (co) variância, por intermédio do método de máxima verossimilhança restrita contra distribuição de Qui-quadrado, com graus de liberdade variando de 1 a 2 , dependendo da diferença no número de efeitos incluídos nos modelos a serem comparados, a $1 \%$ de significância. Somente o modelo MTV não pôde ser comparado com o modelo MA3 por estes possuírem o mesmo número de efeitos incluídos, o que resultaria em zero graus de liberdade.

Em relação aos valores das DEPs, as comparações foram feitas visualmente, sobrepondo o ranking dos 20 melhores touros de cada modelo e verificando se os 20 presentes em um modelo foram os mesmos presentes no outro, o que fornece a idéia de aproximação da classificação dos animais em cada modelo. Esta análise foi feita em um maior conjunto de dados para cada modelo, porém serão descritos apenas 20 para facilitar a configuração da tabela. 
1344 Rev. bras. zootec.

Vale salientar que os resultados desta comparação descrita foram semelhantes aos encontrados no conjunto maior de dados.

Além disso, foram também estimadas a correlações entre as DEPs dos modelos que se apresentaram mais semelhantes ao MA3 na comparação dos 20 primeiros.

As comparações nos componentes de (co) variância foram feitas sempre entre o modelo mais completo (MA3) e os demais. Procedimento semelhante foi aplicado para as DEPs, porém os modelos touro (MT1 e MT2) não puderam ser comparados com o MA3, por resultarem a DEP dos touros, e não dos animais.

\section{Resultados e Discussão}

As estimativas de componentes de (co) variância da característica ganho de peso do nascer à desmama (GPND), para cada modelo, são mostradas na Tabela 2. Os resultados mostraram que, enquanto presente pelo menos um dos efeitos (ambiental ou materno) no modelo, não houve diferença significativa, quando comparados ao modelo animal MA3.

Os modelos MA1 e MA2 resultaram em valores de variância direta muito semelhantes $(81,27$ e 81,76, respectivamente) àquele obtido pelo MA3 $(81,46)$. No entanto, foram observadas diferenças nas estimativas de variância ambiental e materna, quando esses efeitos foram incluídos separadamente no modelo, ou seja, 158,89 para variância materna no MA1 contra 77,85 do MA3, e 119,69 para variância ambiental no MA2 contra 93,42 do MA3.

As herdabilidades $\left(\mathrm{h}^{2}\right)$ diretas foram idênticas para os três modelos $(0,13)$, havendo diferença na $\mathrm{h}^{2}$ materna, que aumentou de 0,13 (MA3) para 0,26 (MA1).

O modelo touro MT1 também resultou em valores semelhantes para $\mathrm{h}^{2}$ direta $(0,12)$ e apresentou valor mais baixo para variância direta $(20,49)$, compensado pelo valor mais alto da variância ambiental $(140,10)$, contra 93,42 do MA3.

Os modelos que não incluíram os efeitos ambiental e materno (MA4 e MT2) mostraram-se estatisticamente $(\mathrm{P}<0,01)$ diferentes em relação ao MA3. É interessante ressaltar que os resultados destes dois modelos também não foram semelhantes entre si, já que, enquanto o modelo animal apresentou valores altos para a variância direta $(244,74)$ e $h^{2}$ direta $(0,38)$, o modelo touro mostrou valores baixos para a variância direta $(19,60) \mathrm{e} \mathrm{h}^{2}$ direta $(0,12)$.

$\mathrm{O}$ modelo MTV, que não pôde ser comparado estatisticamente, não apresentou valores próximos dos encontrados no MA3, porém essa discrepância aparente não foi maior do que aquela verificada entre o modelo touro MT1 e o modelo animal MA3, e esses, por sua vez, não se apresentaram diferentes estatisticamente $(\mathrm{P}>0,05)$.

É interessante ressaltar que a variância direta e

Tabela 2 - Estimativas de (co) variância para a característica ganho de peso do nascer à desmama Table 2 - (Co) variance estimate for weigh gain from birth to weaning

\begin{tabular}{lrrrrrrrrr}
\hline & $\sigma^{2}{ }_{\mathrm{a}}$ & $\sigma_{\mathrm{m}}{ }^{2}$ & \multicolumn{1}{c}{$\sigma_{\mathrm{am}}$} & $\sigma^{2}{ }_{\mathrm{p}}$ & $\mathrm{h}^{2}{ }_{\mathrm{a}}$ & $\mathrm{h}^{2}{ }_{\mathrm{m}}$ & $\mathrm{r}_{\mathrm{am}}$ & $\sigma_{\mathrm{e}}^{2}$ & $-2 \log \mathrm{L}$ \\
\hline MA1 & 81,27 & 158,89 & $-39,46$ & & 0,13 & 0,26 & $-0,35$ & 418,32 & 34374,97 \\
MA2 & 81,76 & & & 119,69 & 0,13 & & & 417,67 & 34374,97 \\
MA3 & 81,46 & 77,85 & $-51,36$ & 93,42 & 0,13 & 0,13 & $-0,64$ & 418,09 & 34374,97 \\
MA4 & 244,74 & & & & 0,38 & & & 400,35 & 34418,39 \\
MTV & 20,36 & 124,81 & $-49,17$ & 15,33 & 0,13 & 0,39 & $-0,98$ & 458,67 & 34374,97 \\
MT1 & 20,49 & & & 140,10 & 0,12 & & & 458,68 & 34374,97 \\
MT2 & 19,60 & & & & 0,12 & & & 598,87 & 34456,65 \\
\hline
\end{tabular}

MA1: modelo animal com efeito aditivo direto do animal e efeito genético materno; MA2: modelo animal com efeito aditivo direto do animal e efeito de ambiente permanente; MA3: modelo animal com os três efeitos já citados; MA4: modelo animal com apenas efeito aditivo direto do animal; MTV: modelo touro-vaca com efeito aditivo direto do pai, efeito genético materno e efeito de ambiente permanente; MT1: modelo touro com efeito aditivo direto do pai e efeito de ambiente permanente; MT2: modelo touro com efeito aditivo direto do pai; $\sigma^{2}$ : variância genética aditiva direta; $\sigma^{2}$ : variância genética aditiva materna; $\sigma_{\text {am }}$ : covariância genética entre efeito direto e efeito materno; $\sigma_{p}^{2}$ : variância de ambiente permanente; $\sigma^{2}$ : variância residual; $h^{2}$ : herdabilidade do efeito direto; $h_{m}^{2}$ : herdabilidade do efeito materno; $r_{a m}$ : correlação genética entre efeito direto $e$ efeito materno.

MA1: animal model with direct additive genetic effect of the animal and with maternal effect; MA2: animal mode/ with direct additive genetic effect and with permanent environmental effect; MA3: animal model with the three effects; MA4: animal model with only the directgenetic effect of the animal; MTV: sire-dam model with direct genetic effect of sire, maternal effect and permanent environmental effect; MT1: sire model with direct additive genetic effect of sire and permanent environmental effect; MT2: sire model with the genetic direct effect of sire; $\sigma_{a}^{2}$ : additive genetic variance; $\sigma_{m}^{2}$ : additive genetic maternal variance; $\sigma_{a m}$ : genetic covariance between direct and maternal effect; $\sigma_{p}^{2}$ : permanent environmental variance; $\sigma_{e}^{2}$ : residual variance; $h_{a}^{2}$ : direct heritability; $h_{m}^{2}$ : maternal heritability; $r_{a m}$ : genetic correlation between direct and maternal effect. 
a $\mathrm{h}^{2}$ direta mantiveram os seus valores nos modelos que incluíram pelo menos um dos efeitos (ambiental ou materno).

A covariância genética entre efeito direto e materno foi bastante semelhante nos modelos que incluíram o efeito materno, sendo -39,46, -51,36 e -49,17 para o MA1, MA3 e MTV, respectivamente. Entretanto, a correlação entre as $\mathrm{h}^{2}$ direta e materna não apresentou a mesma variação, e os valores obtidos resultaram em estimativa superestimada para o MTV $(-0,98)$ e subestimada para o MA1 (-0,35), quando comparado ao MA3 $(-0,64)$.

As estimativas de DEPs para esta característica (Tabela 5), quando analisadas em relação à sobreposição do ranking dos 20 primeiros animais, mostraram-se semelhantes, quando se compararam o MA1 e o MA3 (100\%). Entretanto, em relação à comparação entre MA2 e MA3 (55\%), MA4 e MA3 $(10 \%)$ e MTV e MA3 (20\%), estes se portaram desiguais, com exceção do MA2, que, ao ser observado em todo o grupo, não foi diferente.

Na correlação (Tabela 6), tanto para o modelo MA1 como para o MA2, em relação ao MA3, esta estimativa foi alta e significativa, de 0,99733 e de 0,99164, respectivamente.

Para a característica ganho de peso pós-desmama (GPPD), cujos resultados estão na Tabela 3, com exceção do MTV, que, apesar de não poder ser comparado por este método, o qual, entretanto, tam- bém se mostrou visualmente semelhante, os demais modelos não apresentaram diferença estatística em relação ao MA3, nem mesmo os que não incluíram ambos os efeitos já citados. Este resultado já era esperado, devido à menor influência da mãe para características pós-desmama.

Observa-se que os modelos touro e touro-vaca, que não incluem efeito aditivo direto do animal, subestimaram os valores da variância direta (o que também foi observado na primeira característica), sendo que os valores decrescem cerca de $70 \%$.

Os valores da variância materna foram mais próximos entre os modelos animal, que incluíram este efeito, do que para o modelo MTV (34,36 para o MA1 e 50,37 para o MA3, contra 16,57 para o MTV). Já a variância ambiental resultou em valores baixos, o que mais uma vez salienta a baixa influência das características da mãe no pós-desmame.

A covariância genética entre os efeitos direto e materno foi semelhante nos modelos animal $(-39,06 \mathrm{e}$ $-54,96$ para MA1 e MA3, respectivamente), enquanto o MTV mostrou um valor subestimado de $-18,46$.

As $\mathrm{h}^{2}$ diretas foram muito semelhantes para todos os modelos, ao passo que a $\mathrm{h}^{2}$ materna ficou superestimada no modelo MTV $(0,32)$, havendo novamente menor diferença entre os modelos animal $(0,22$ do MA3 e 0,15 do MA1). Embora não tenha ocorrido diferença significativa entre os modelos estudados, os modelos animal mostraram-se mais semelhantes.

Tabela 3 - Estimativas de (co) variância para a característica ganho de peso pós-desmama Table 3 - (Co) variance estimates for post weaning weight gain trait

\begin{tabular}{lccccccccc}
\hline & $\sigma^{2}{ }_{\mathrm{a}}$ & $\sigma_{\mathrm{m}}{ }^{2}$ & $\sigma_{\mathrm{am}}$ & $\sigma_{\mathrm{p}}$ & $\mathrm{h}^{2}{ }_{\mathrm{a}}$ & $\mathrm{h}^{2}{ }_{\mathrm{m}}$ & $\mathrm{r}_{\mathrm{am}}$ & $\sigma_{\mathrm{e}}$ & $-2 \log \mathrm{L}$ \\
\hline MA1 & 84,89 & 34,36 & $-39,06$ & & 0,37 & 0,15 & $-0,72$ & 151,05 & 13557,38 \\
MA2 & 82,31 & & & 0,31 & 0,36 & & & 148,39 & 13557,47 \\
MA3 & 84,92 & 50,37 & $-54,96$ & 0,00075 & 0,37 & 0,22 & $-0,84$ & 150,96 & 13557,38 \\
MA4 & 83,28 & & & & 0,36 & & & 147,86 & 13557,46 \\
MTV & 21,23 & 16,57 & $-18,46$ & 0,000099 & 0,37 & 0,32 & $-0,99$ & 193,46 & 13557,38 \\
MT1 & 21,28 & & & 0,00041 & 0,36 & & & 209,50 & 13558,51 \\
MT2 & 21,35 & & & & 0,36 & & & 209,69 & 13558,51 \\
\hline
\end{tabular}

MA1: modelo animal com efeito aditivo direto do animal e efeito genético materno; MA2: modelo animal com efeito aditivo direto do animal e efeito de ambiente permanente; MA3: modelo animal com os três efeitos já citados; MA4: modelo animal com apenas efeito aditivo direto do animal; MTV: modelo touro-vaca com efeito aditivo direto do pai, efeito genético materno e efeito de ambiente permanente; MT1: modelo touro com efeito aditivo direto do pai e efeito de ambiente permanente; MT2: modelo touro com efeito aditivo direto do pai; $\sigma^{2}$ : variância genética aditiva direta; $\sigma^{2}$ : variância genética aditiva materna; $\sigma_{a m}$ : covariância genética entre efeito direto e efeito materno; $\sigma^{2}$ : variância de ambiente permanente; $\sigma_{\mathrm{e}}^{2}$ : variância residual; $\mathrm{h}_{\mathrm{a}}{ }_{\mathrm{a}}$ : herdabilidade do efeito direto; $\mathrm{h}_{\mathrm{m}}^{2}$ : herdabilidade do efeito materno; $r_{\text {am: }}$ : correlação genética entre efeito direto e efeito materno.

MA1: animal model with direct additive genetic effect of the animal and with maternal effect; MA2: animal model with direct additive genetic effect and with permanent environmental effect; MA3: animal model with the three effects; MA4: animal model with only the direct genetic effect of the animal; MTV: sire-dam model with direct genetic effect of sire, maternal effect and permanent environmental effect; MT1: sire model with direct additive genetic effect of sire and permanentenvironmental effect; $M$ T2: sire model with the genetic direct effect of sire; $\sigma^{2}$ : additive genetic variance; $\sigma_{m}^{2}$ : additive genetic maternal variance; $\mathrm{s}_{\text {am }}$ : genetic covariance between direct and maternal effect; $\sigma_{p}^{2}$ : permanent environmental variance; $\sigma^{2}{ }_{e}$ : residual variance; $h_{a}^{2}$ : direct heritability; $h^{2}{ }_{m}:$ maternal heritability; $r_{\text {am }}$ : genetic correlation between direct and maternal effect. 
1346 Rev. bras. zootec.

Já as estimativas de DEP, para esta segunda característica (Tabela 5), apresentaram valores semelhantes em relação ao MA3, os modelos MA1 (95\%), MA2 (80\%) e MA4 (80\%), sendo que apenas o modelo MTV se mostrou diferente, com apenas $5 \%$ de igualdade ao MA3 nestes vinte animais.

A correlação para esta característica (Tabela 6) também apresentou-se alta e significativa, de 0,98447 (MA1 e MA3) e 0,99565 (MA2 e MA3).

$\mathrm{Na}$ análise bivariada (Tabela 4 ) realizada utilizando os mesmos sete modelos, observou-se, tanto para a primeira quanto para a segunda característica, semelhança em relação à analise univariada apenas na estimativa de valores da $\sigma^{2}$ direta e $h^{2}$ direta.

Vale salientar que alguns valores foram inesperados, como a covariância entre os efeitos direto e materno para a primeira característica no modelo MA1, que foi negativa $(-39,46)$ na univariada e apresentou, na análise bivariada, valor positivo $(1,23)$; além de valores que resultaram em zero, como a correlação entre os efeitos direto e materno para a segunda característica no modelo MA1, o que pode ser atribuído ao pequeno número de animais.

Comparando estatisticamente os modelos por esta análise, os resultados foram semelhantes ao encontrado na univariada para o GPND, apresentando diferença apenas dos modelos que não incluíram pelo menos um dos efeitos (ambiental ou materno), em relação ao modelo animal MA3.

A diferença na maioria das estimativas é que torna interessante a realização desta análise para fins de comparação com os resultados da análise univariada.

Tabela 4 - Análise bivariada para estimativas de (co) variância para as características ganho de peso do nascer à desmama e ganho de peso pós-desmama

Table 4 - Bivariate analysis for (co) variance estimate for weight gain from birth to weaning and post-weaning weight gain traits

\begin{tabular}{|c|c|c|c|c|c|c|c|c|c|c|}
\hline & $\sigma_{\mathrm{a}}^{2}$ & $\sigma_{\mathrm{m}}^{2}$ & $\sigma_{\mathrm{am}}$ & $\sigma_{p}^{2}$ & $\mathrm{~h}^{2}{ }_{\mathrm{a}}$ & $\mathrm{h}^{2}{ }_{\mathrm{m}}$ & $\mathrm{r}_{\mathrm{am}}$ & $\sigma_{\mathrm{e}}^{2}$ & $\mathrm{r}_{\mathrm{ala} 2}$ & $-2 \log \mathrm{L}$ \\
\hline$\overline{\mathrm{MA} 1}$ & 77,83 & 120,4 & 1,23 & & 0,13 & 0,19 & 0,01 & 420,08 & $-0,19$ & 47931,44 \\
\hline MA1 & 78,99 & 10,51 & 0,0035 & & 0,34 & 0,05 & 0,00 & 141,84 & & \\
\hline MA2 & 81,52 & & & 119,68 & 0,13 & & & 418,27 & $-0,15$ & 47930,71 \\
\hline MA2 & 82,80 & & & 0,92 & 0,36 & & & 147,38 & & \\
\hline MA3 & 81,90 & 119,23 & $-0,04$ & 0,000018 & 0,13 & 0,19 & 0,00 & 418,27 & $-0,17$ & 47930,50 \\
\hline MA3 & 87,89 & 11,84 & $-24,85$ & 6,87 & 0,38 & 0,05 & $-0,77$ & 149,88 & & \\
\hline MA4 & 244,36 & & & & 0,38 & & & 400,24 & $-0,01$ & 47975,60 \\
\hline MA4 & 83,04 & & & & 0,36 & & & 147,93 & & \\
\hline MTV & 20,62 & 139,91 & 0,18 & 0,19 & 0,13 & 0,26 & $-0,36$ & 458,79 & I & 47931,38 \\
\hline MTV & 21,66 & 16,37 & $-5,85$ & 0,017 & 0,37 & 0,21 & $-0,84$ & 193,51 & & \\
\hline MT1 & 20,44 & & & 139,72 & 0,12 & & & 459,16 & $-0,17$ & 47930,50 \\
\hline MT1 & 21,89 & & & 15,87 & 0,36 & & & 193,73 & & \\
\hline MT2 & 19,83 & & & & 0,12 & & & 598,95 & $-0,19$ & 48014,42 \\
\hline MT2 & 21,76 & & & & 0,36 & & & 209,56 & & \\
\hline
\end{tabular}

MA1: modelo animal com efeito aditivo direto do animal e efeito genético materno; MA2: modelo animal com efeito aditivo direto do animal e efeito de ambiente permanente; MA3: modelo animal com os três efeitos já citados; MA4: modelo animal com apenas efeito aditivo direto do animal; MTV: modelo touro-vaca com efeito aditivo direto do pai, efeito genético materno e efeito de ambiente permanente; MT1: modelo touro com efeito aditivo direto do pai e efeito de ambiente permanente; MT2: modelo touro com efeito aditivo direto do pai; $\sigma^{2}{ }_{\mathrm{a}}$ : variância genética aditiva direta; $\sigma_{m}^{2}$ : variância genética aditiva materna; $\sigma_{a m}$ : covariância genética entre efeito direto e efeito materno; $\sigma^{2}$ : variância de ambiente permanente; $\sigma_{\mathrm{e}}^{2}$ : variância residual; $\mathrm{h}_{\mathrm{a}}^{2}$ : herdabilidade do efeito direto; $\mathrm{h}_{\mathrm{m}}^{2}$ : herdabilidade do efeito materno; $\mathrm{r}_{\mathrm{am}}$ : correlação genética entre efeito direto e efeito materno; $r_{\mathrm{a} 1} \mathrm{a} 2$ : correlação genética entre ganho de peso do nascer à desmama e ganho de peso pós-desmama.

MA1: animal model with direct additive genetic effect of the animal and with maternal effect; MA2: animal model with direct additive genetic effect and with permanent environmental effect; MA3: animal model with the three effects; MA4: animal model with only the direct genetic effect of the animal; MTV: sire-dam model with directgenetic effect of sire, maternal effect and permanentenvironmental effect; MT1: sire mode/ with directadditive genetic effect of sire and permanentenvironmental effect; MT2: sire mode/ with the genetic direct effect of sire; ${ }^{2}$ : additive genetic variance; $\sigma_{m}^{2}$ : additive genetic maternal variance; $\sigma_{a m}$ : genetic covariance between direct and maternal effect; $\sigma_{p}^{2}$ : permanent environmental variance; $\sigma_{e}^{2}:$ residual variance; $h_{a}^{2}:$ direct heritability; $h_{m}^{2}:$ maternal heritability; $r_{\text {am }}$ : genetic correlation between direct and maternal effect; $r_{\text {a1a2 }}$ : genetic correlation between weight gain from birth to weaning and weight gain post weaning. 
Tabela 5 - Estimativas de DEP (diferença esperada na progênie) para as características ganho de peso do nascer à desmama e pós-desmama, para os vinte animais melhor classificados

Table - EPD (expected breeding value) estimate for weight gain from birth to weaning and weight gain post weaning traits of the twenty best animals

\begin{tabular}{lccrcc}
\hline & \multicolumn{2}{c}{ GPND } & & \multicolumn{2}{c}{ GPPD } \\
\hline & \multicolumn{2}{c}{$\begin{array}{c}\text { Vinte melhores } \\
\text { Best twenties }\end{array}$} & & \multicolumn{2}{c}{$\begin{array}{c}\text { Vinte melhores } \\
\text { Best twenties }\end{array}$} \\
\cline { 2 - 3 } \cline { 5 - 6 } & $\begin{array}{c}\text { Número } \\
\text { Number }\end{array}$ & $\begin{array}{c}\text { Percentagem } \\
\text { Percentage }\end{array}$ & & $\begin{array}{c}\text { Número } \\
\text { Number }\end{array}$ & $\begin{array}{c}\text { Percentagem } \\
\text { Percentage }\end{array}$ \\
\hline MA3 - MA1 & 20 & $100 \%$ & & 19 & $95 \%$ \\
MA3 - MA2 & 11 & $55 \%$ & & 16 & $80 \%$ \\
MA3 - MA4 & 02 & $10 \%$ & & 16 & $80 \%$ \\
MA3 - MTV & 04 & $20 \%$ & & 01 & $5 \%$
\end{tabular}

GPND: ganho de peso do nascer à desmama (Weight gain from birth to weaning).

GPPD: ganho de peso pós desmama (Post-weaning weight gain).

Tabela 5 - Correlação entre as estimativas de DEP (diferença esperada na progênie) para as características ganho de peso do nascer à desmama e pós-desmama

Table 5 - Correlation between EPD (expected breeding value) estimate for weight gain from birth to weaning and weight gain post weaning traits

\begin{tabular}{lcc}
\hline & GPND & GPPD \\
\cline { 2 - 3 } MA3 - MA1 & 0,99733 & 0,98447 \\
MA3 - MA2 & 0,99164 & 0,99565 \\
\hline GPND: ganho de peso do nascer à desmama (Weight gain from birth to \\
weaning). \\
GPPD: ganho de peso pós desmama (Post-weaning weight gain).
\end{tabular}

\section{Conclusões}

Os resultados de componentes de (co) variância indicam que, para características pré-desmame, a importância da inclusão de efeitos referentes ao efeito materno, bem como ao ambiente permanente, representado neste caso pela habilidade leiteira da mãe, é de maior importância, sendo necessária a inclusão de pelo menos um destes efeitos no modelo para estimar o valor genético dos animais em estudo, sem alterar significativamente o seu componente genético aditivo direto, sendo que os modelos MA1, MA2 e MT1 podem ser utilizados em substituição ao modelo MA3.

Porém, para o pós-desmame, em que foi observada menor influência das características da vaca sobre o desempenho do indivíduo, modelos que não incluíram os efeitos materno e ambiental permanente também mostraram-se sem diferença estatística, sendo que os modelos MA4 e MT2 podem ser usados em substituição ao MA3, assim como os demais modelos já citados.

O modelo MTV, que não pôde ser comparado estatisticamente ao MA3, apresentou variação semelhante aos demais modelos e, como incluiu ambos os efeitos citados, provavelmente pode ser utilizado em substituição ao MA3.

Para cálculo de DEP, pode-se sugerir, pelos resultados obtidos, que, para ambas as características, os modelos MA1 e MA2 podem substituir o modelo MA3, sendo o MA1 o mais indicado para a primeira característica (GPND).

\section{Referências Bibliográficas}

BERTRAND, J.K., BENYSHEK, L.L. 1987. Variance and covariance for maternally influenced beef growth traits. $J$. Anim. Sci., 64:728.

BOLDMAN, K.G., KRIESE, L.A., VAN VLECK, L.D. et al. 1995. A manual for use of MTDFREML: a set of programs to obtain estimates of variance and covariance. Lincoln: Department of Agriculture/ Agriculture Research Service. $120 \mathrm{p}$.

DICKERSON, G.E. 1947. Composition of hog carcasses as influenced by heritable differences in rate and economy of gain. Iowa Agric. Exp. Stn. Res. Bull. 354p.

FERREIRA, G.B., MacNEIL, M.D., Van VLECK, L.D. 1999. Variance components and breeding values for growth traits from different statistical models. J. Anim. Sci., 77:26412650.

HENDERSON, C.R. 1976. A simple method for computing the inverse of a numerator relationship matrix used in prediction of breeding values. Biometrics, 32:69.

JOHNSON, Z.B., WRIGHT, D.W., BROWN, C.J. et al. 1992. Effect of including relationship in the estimation of genetic parameters of beef calves. J. Anim. Sci., 70:78.

KOCH, R.M., CLARK, R.I. 1955. Genetic and environmental 
1348 Rev. bras. zootec.

relationships among economic characters in beef cattle. III. Evaluating maternal environment. J. Anim. Sci., 14:979.

KOOTS, K.R., GIBSON, J.P., WILTON, J.W. 1994. Analyses of published genetic parameter estimates for beef production traits. 1. Heritability. Anim. Breed. Abst., 62:825.

KOOTS, K.R., GIBSON, J.P., WILTON, J.W. 1994. Analyses of published genetic parameters estimates for beef production traits. 2. Phenotypic and genetic correlation. Anim. Breed. Abst., 62:825.

MERCADANTE, M.E.Z., LÔBO, R.B. 1997. Estimativas de (co) variâncias e parâmetros genéticos dos efeitos direto e materno de características de crescimento de fêmeas de um rebanho Nelore. R. Bras. Zootec., 26(6):1124-1133.

MEYER, K., CARRICK, M.J., DONNELLY, B.J.P. 1993. Genetic parameters for growth traits of Australian beef cattle from a multibreed selection experiment. J. Anim. Sci., 71(10):2614-2622.

MOHIUDDIN, G. 1993. Estimates of genetic phenotypic parameters of some performance traits in beef cattle. Anim. Breed. Abst., 61:495.
SWALVE, H.H. 1993. Estimation of direct and maternal (co) variance components for growth traits in Australian Simmental beef cattle. J. Anim. Breed. Genet., 110:241.

THOMPSON, R. 1976. The estimation of maternal genetic variances. Biometrics, 32:903.

VAN VLECK, L.D., HUDSON, G.F.S. 1982. Relationship among sires in estimating genetic variances. J. Dairy Sci., 65:1663.

WILHAM, R.L. 1963. The covariance between relatives for characters composed of components contributed by related individuals. Biometrics, 19:18.

WILHAM, R.L. 1972. The role of maternal effects in animal breeding: III. Biometrical aspects of maternal effects in animals. J. Anim. Sci., 35:1288.

Recebido em: 25/08/99

Aceito em: 16/03/00 